\title{
Philosophiques
}

\section{Relations de vérifaction}

\section{Étude critique de : E. J. Lowe et A. Rami (dir.), Truth and Truth-Making, Stocksfield, Acumen, 2009, 262 p.}

\section{Anne-Marie Boisvert}

Volume 38, numéro 1, printemps 2011

URI : https://id.erudit.org/iderudit/1005731ar

DOI : https://doi.org/10.7202/1005731ar

Aller au sommaire du numéro

Éditeur(s)

Société de philosophie du Québec

ISSN

0316-2923 (imprimé)

1492-1391 (numérique)

Découvrir la revue

Citer cet article

Boisvert, A.-M. (2011). Relations de vérifaction / Étude critique de : E. J. Lowe et

A. Rami (dir.), Truth and Truth-Making, Stocksfield, Acumen, 2009, 262 p.

Philosophiques, 38(1), 305-330. https://doi.org/10.7202/1005731ar d'utilisation que vous pouvez consulter en ligne.

https://apropos.erudit.org/fr/usagers/politique-dutilisation/ 


\title{
Étude critique
}

\section{Relations de vérifaction}

\author{
Étude critique de: E. J. Lowe et A. Rami (dir.), Truth and Truth-Making, \\ Stocksfield, Acumen, 2009, 262 p.
}

\author{
ANNE-MARIE BOISVERT \\ Université du Québec à Montréal
}

\section{La vérifaction}

L'intuition selon laquelle la vérité dépend de la réalité est une intuition puissante et tenace. Les questions surgissent cependant dès qu'on cherche à préciser les modalités de cette relation. En métaphysique contemporaine, les discussions sur ce thème se sont plus particulièrement centrées sur la notion de vérifaction depuis un certain nombre d'années déjà. Les vérifactionnistes — David Armstrong en tête - entendent préserver l'intuition à la base de la conception correspondantiste de la vérité tout en évitant les écueils de cette dernière, notamment en ne s'encombrant pas du «dogme de la correspondance terme à terme» (voir Armstrong 2004c, p. 105). Ils soutiennent que la vérité d'un vériporteur (c'est-à-dire d'un énoncé ou, comme on l'admet plus généralement, d'une proposition) doit être nécessitée par l'existence d'au moins une entité dans le monde. La relation de vérifaction est ainsi conçue, dans sa version orthodoxe, comme une relation existentielle interne, dans laquelle l'existence d'un vériporteur et celle de son ou ses vérifacteurs suffisent pour que ledit vériporteur soit vrai. En retour, la qualité de vérifacteur conférée à une entité constitue selon les vérifactionnistes une bonne raison d'admettre celle-ci dans notre ontologie. Répondre à la «question de la vérification » reviendrait donc à se prononcer sur l'ameublement du monde:

[P]ostuler certains vérifacteurs pour certaines vérités, c'est admettre ces vérifacteurs dans son ontologie (Armstrong, 2004b, p. 131).

En ce sens, la théorie de la vérifaction se pose en rivale du critère quinien de l'engagement ontologique limité aux référents des seules expressions en position de sujet ${ }^{1}$. Mais, rétorquent les critiques du vérifactionnisme, que quelque chose dans le monde «rende vraie» une vérité signifie-t-il obligatoi-

1. «Demander des vérifacteurs peut être une meilleure question que celle de Quine», Armstrong 2010, p. 64. Armstrong parle à ce sujet de «justice ontologique»: "[à] mes yeux, le prédicat peut et doit régulièrement porter un engagement ontologique, et l'un des avantages que je vois à postuler des états de choses comme vérifacteurs est que nombre de prédicats, au lieu de refléter une pure «idéologie» comme le dit Quine, s'appliquent en vertu des caractéristiques objectives des choses» (Armstrong 2004d, p. 133). Voir aussi Armstrong 1989, p. 9-10; Beebee et Dodd 2005, p. 2-3; Hochberg 2009, p. 158-161. 
rement qu'il s'agisse de quelque chose? Car même s'il y a pour toute vérité un vérifacteur, "il ne s'ensuit pas que le vérifacteur en question existe» (Daly, 2005, p. 86, l'italique est de moi). Il peut s'agir d'entités meinongiennes (voir ibid.); ou encore, la vérité peut dépendre, non de l'existence des choses, mais de la manière dont sont les choses (non pas «whether things are ", mais "how things are $»^{2}$ ). Sans compter que la version maximaliste du principe de vérifaction, qui prétend étendre ledit principe à toutes les vérités, y compris les vérités nécessaires, générales et négatives, risque de conduire à l'admission d'entités fort baroques, telles que les faits négatifs ou disjonctifs ${ }^{3}$. Une stratégie a été d'abandonner le maximalisme. Il demeure que les vérifactionnistes doivent au minimum fournir des vérifacteurs pour les propositions atomiques vraies de manière contingente ${ }^{4}$. Et cette stratégie ne satisfera pas ceux qui s'opposent à l'admission des faits tout court (des faits positifs), des états de choses, ou même des tropes, dans l'ontologie, parce qu'ils les considèrent de toute façon comme des entités superflues, produits de réifications indues. On pourrait même soutenir qu'une telle stratégie (celle de restreindre la portée du principe de vérifaction) revient en fait à fournir des munitions aux opposants de ce principe: si celui-ci ne doit pas s'appliquer à certaines vérités, telles les vérités négatives, pourquoi devrait-il valoir pour les vérités positives? "Par conséquent, ou bien le maximalisme est vrai, ou bien nous devons abandonner la théorie de la vérifaction dans son ensemble» (Cameron, 2010, p. 412).

L'autre problème majeur est celui de la nature de la relation de vérifaction en tant que telle: qu'est-ce au juste que cette relation de nécessitation? Comprise comme une implication classique, elle entraîne des conséquences qui trivialisent à outrance le principe en question (voir les critiques de Restall, 1996, sur lesquelles je reviendrai). D'ailleurs, la relation de vérifaction étant transcatégorielle, l'analyse en termes d'implication serait incorrecte à proprement parler (voir Armstrong, 2004d, p. 130, et Armstrong, 2004b, p. 116). Pourrait-on tenter d'expliquer plus judicieusement cette relation en termes de fondation (grounding) (Rodriguez-Pereyra) ou de dépendance existentielle (Mulligan et al), d'essence (Mulligan, Lowe) ou d'intrinsicalité (Parsons) ? Bref, les débats font rage, et la vérifaction constitue assurément un sujet chaud à l'heure actuelle en philosophie contemporaine. En témoigne la parution de nombreux articles et ouvrages, ainsi que celle de trois recueils majeurs consacrés à cette question: Truthmakers: The Contemporary Debate (2005), sous la direction de H. Beebee et J. Dodd, Metaphysics and

2. Voir en particulier les travaux de Lewis et de Dodd.

3. Ou encore le fameux état de choses totalisant d'Armstrong, que d'aucuns ont surnommé le «Porky the Pig fact» («That's all folks»...) (voir Forrest et Khlentzos, 2000, p. 7).

4. «Les propositions atomiques vraies de manière contingente sont ce pour quoi il doit y avoir des vérifacteurs s'il y a bel et bien des vérifacteurs» (Simons et Melia, 2000, p. 66; voir aussi Rodriguez-Pereyra, 2005). 
Truthmakers (2007), sous la direction de J.-M. Monnoyer et enfin, Truth and Truth-Making (2009), sous la direction d'E. J. Lowe et d'A. Rami, qui m'occupera plus particulièrement ici ${ }^{5}$.

\section{Le recueil de Lowe et Rami}

Le recueil de Lowe et Rami est divisé en deux parties. La première, intitulée "Setting the Stage», rassemble quelques articles devenus des «classiques» de la théorie de la vérifaction ${ }^{6}$, précédés d'un article panoramique et historique de Kevin Mulligan portant sur les origines du principe de vérifaction en tant que tel. La deuxième partie, intitulée "The Current Debate", propose des articles plus récents, dont quatre inédits, avec pour but de situer le lecteur au cœur des débats actuels concernant la vérifaction et la vérité. L'introduction fouillée et savante rédigée par Adolf Rami sert quant à elle à faire le pont entre les deux parties, en résumant bien les enjeux et les problèmes posés tant par la version orthodoxe de la théorie de la vérifaction que par ses diverses versions de rechange.

On a reproché au recueil de Lowe et Rami la chose suivante:

Le problème principal de cette anthologie est qu'elle se donne pour objectif d'être à la fois un livre de lecture de base sur la théorie de la vérifaction $e t$ une collection de nouveaux essais sur la théorie de la vérifaction, tout cela en deux cent soixante-deux pages. Elle n'accomplit bien aucun de ces buts en cherchant à les remplir tous les deux en même temps (Efird, 2009; voir aussi Dodd, 2010).

Certes, en tant qu'ouvrage introductif ou manuel, le recueil de Lowe et Rami est à la fois trop restreint et trop pointu. Ainsi, après avoir parcouru un certain nombre d'articles dans le domaine et acquis une certaine connaissance de la théorie de la vérifaction et de ses enjeux, on lira l' "introduction » de Rami comme un (excellent) récapitulatif, avec davantage de profit qu'avant. Et on peut s'interroger sur le choix et la présentation des articles de la première section: le texte de Mulligan, bien qu'intéressant, n'est pas un texte «majeur» de son auteur et demeure par ses considérations avant tout historiques en marge du débat actuel; il aurait été intéressant de proposer à la place (étant donné la brièveté du recueil) un texte ou un extrait d'un ouvrage de Bigelow, par exemple, puisque la question de la survenance comme autre solution à la version orthodoxe de la vérifaction a été et

5. Mentionnons en outre l'excellent numéro de Logique et analyse consacré à la vérifaction (vol. 43, n 169-170, 2000), malheureusement épuisé, ainsi que le recueil paru en 2004 sous la direction de J.-M. Monnoyer, La structure du monde, qui comporte toute une section sur cette question (avec des textes d'Armstrong, de Horwich et d'autres).

6. Ces articles sont: "Truth-Makers», de K. Mulligan, P. Simons et B. Smith (1984); «Truth-Makers, Entailment and Necessity », de G. Restall (1996); "Truth-Making and Difference-Making", de D. Lewis (2001); "The General Theory of Truth-Making», de D. M. Armstrong (chapitre 2 de son ouvrage Truth and Truthmakers (2004)). 
continue d'être largement débattue. Les contributions de Restall, de Lewis et d'Armstrong au débat sur la vérifaction sont certes des incontournables, et leur inclusion dans le recueil est la bienvenue, d'autant plus que le texte de Restall est suivi d'une postface inédite. Mais pour un recueil censé "rassembler les articles les plus importants sur la vérifaction des trois dernières décennies" (dixit la quatrième de couverture), la sélection est plutôt mince. Et en ajoutant à cette sélection réduite de textes «classiques » une collection d'articles plus récents mais pas forcément inédits, le recueil donne à première vue une impression d'éparpillement en même temps que de redite par rapport aux recueils précédemment parus de Monnoyer, et de Beebee et Dodd (certains des textes réunis dans Lowe et Rami ayant déjà été publiés - ou republiés - dans l'un ou l'autre de ces recueils $)^{7}$. En comparaison, ceux-ci paraissent davantage unifiés. Le premier rassemble en effet les communications présentées à l'occasion d'une conférence intitulée "Truths and Truthmakers: Vingt ans après ${ }^{8}$ et organisée pour souligner deux dates marquantes dans l'histoire de la vérifaction: 1984, parution du texte de Mulligan, Simons et Smith déjà mentionné; 2004, parution de l'ouvrage de David Armstrong, Truth and Truthmakers. Monnoyer profite de l'occasion pour republier pour la première fois depuis 1984 le texte de Mulligan, Simons et Smith (avant sa re-republication dans le recueil de Rami et Lowe, laquelle paraît du coup bien moins pertinente), en plus de présenter les nouvelles contributions au débat de Mulligan, de Simons et de Smith eux-mêmes qui ont poursuivi au cours des années, chacun de leur côté, leur réflexion sur la question de la vérifaction. En outre, Stefano Caputo offre dans le même recueil une critique pénétrante des thèses plus récentes de Mulligan et de Smith. Monnoyer (2007) bénéficie enfin de la contribution de David Armstrong, dont la présence à ce colloque a donné lieu à des échanges fructueux sur son ouvrage et ses thèses avec plusieurs participants, notamment Philipp Keller. Le recueil de Beebee et Dodd propose pour sa part une excellente introduction (rédigée par ses deux directeurs) sans doute moins minutieusement détaillée que celle de Rami, mais qui réussit à résumer les principaux enjeux à la base du débat de manière brève et claire en même temps qu'enga-

7. Les textes inédits dans Lowe et Rami se résument donc, stricto sensu, à l'introduction de Rami, aux textes de David, Hochberg et Parsons, et aux postfaces de Restall et RodriguezPereyra. Le texte de Horwich, présenté comme inédit, est en fait déjà paru en 2008 dans Midwest Studies in Philosophy, XXXII, p. 258-273 (notons également qu'une première version de ce texte est parue en français dans Monnoyer 2004, dans le cadre d'un échange très intéressant entre Armstrong et Horwich). Le texte de Mulligan a paru en allemand en 2006. Le texte de Lowe, enfin, présenté également comme inédit, est peu ou prou le même texte que celui inclus dans Monnoyer (2007) sous le titre "Truthmaking as Essential Dependence» (ou en constitue à tout le moins une nouvelle version fort semblable).

8. Conférence qui s'est tenue à l'Université d'Aix-en-Provence du 9 au 11 décembre 2004. 
geante. Les articles — tous inédits ${ }^{9}-$ y sont en outre uniformément d'une excellente teneur.

Cela étant dit, le recueil de Lowe et Rami présente un intérêt majeur, celui d'être composé principalement d'articles abordant la question de la nature de la relation de vérifaction en tant que telle sans la reformuler et la réduire, par montée sémantique, à une relation d'implication entre propositions comme le font Beebee et Dodd ${ }^{10}$. Car, comme le remarque John Heil: «[l]es vérificateurs des énoncés, quel que soit le niveau de ceux-ci, sont des manières d'être du monde du premier ordre » («truth-makers for statements at whatever level are first-order ways the world is»), et il ne sert à rien de laisser une proposition jouer le rôle d'un vérifacteur par procuration ( "go proxy for »), puisque cela ne fait que déplacer le problème (voir Heil, 2000, p. 236 et p. 234; voir aussi Fumerton, 2006). Le défi principal qui se pose aux vérifactionnistes, au-delà des problèmes spécifiques posés par le maximalisme, est d'abord de réussir à rendre compte de manière satisfaisante de la relation de vérifaction en tant que relation de nécessitation transcatégorielle (voir Rami, 2009, p. 24, et Caputo, 2007, p. 276). On pourra ainsi lire Lowe et Rami comme un débat sur cette question ${ }^{11}$, entre, d'une part, les critiques du vérifactionnisme (David Lewis et surtout Paul Horwich ${ }^{12}$ ), et d'autre part, ses défenseurs (Kevin Mulligan, Peter Simons, Barry Smith et David Armstrong (bien entendu), E. J. Lowe et Gonzalo Rodriguez-Pereyra). À ce sujet, les contributions de Greg Restall et de Marian David ne doivent pas être oubliées, car elles apportent un éclairage ciblé sur le débat (sur les rapports entre vérifaction et implication logique pour le premier, vérifaction et correspondance pour le second) permettant de commencer à mieux cerner la nature de la relation en question, ce qu'elle est et ce qu'elle n'est pas. La présente étude prendra donc la forme d'une enquête, au cours de laquelle je suivrai ce fil thématique en particulier. Il est évident que, ce faisant, de nombreux développements intéressants sur des questions importantes devront être laissés de côté, ainsi que certains textes moins pertinents pour la question dont j'ai choisi de m'occuper ${ }^{13}$.

9. Issus, pour la plupart, d'une conférence qui a eu lieu en 2002, à l'Université de Manchester.

10. La relation de vérifaction devenant sous leur plume: «(VF) Nécessairement, si $<\mathrm{p}\rangle$ est vraie, alors il y a au moins une entité $a$ telle que $<a$ existe $>$ implique que $<<$ p $>$ est vraie $>$ " (Beebee et Dodd 2005, p. 2).

11. Remarquons en passant que le recueil s'intitule Truth and Truth-Making (plutôt que Truth and Truthmakers, comme chez Armstrong), ce qui met effectivement l'accent sur la relation de vérifaction en tant que telle.

12. Il faudrait ajouter ici les noms de Julian Dodd et Jennifer Hornsby, absents du recueil, mais auxquels (en plus de Lewis) Rodriguez-Pereyra répond dans ses articles.

13. Celui de Josh Parsons, ou celui de Herbert Hochberg (au demeurant remarquable), par exemple. 


\section{3. «Setting the Stage»}

Il faudrait, pour retracer l'histoire de la vérifaction, remonter jusqu'à Aristote (voir Mulligan et al, 1984). Plus récemment, on a pu retrouver des considérations de cet ordre chez certains philosophes de la première moitié du vingtième siècle, entre autres Russell en Angleterre (avec sa notion de «vérifieur» [verifier]), ou Pfänder en Allemagne (voir Mulligan et al, 1984, Mulligan, 2009, Armstrong, 2004a). Armstrong a par ailleurs raconté à maintes reprises les circonstances dans lesquelles C. B. Martin a introduit la notion de vérifaction en tant que telle à la fin des années cinquante comme argument contre le phénoménalisme (voir entre autres Armstrong, 2004a, p. 1-3 et 2010, p. 61), et comment, ce faisant, Martin s'est trouvé à l'origine d'un courant très vivace au sein de ce que l'on a appelé le réalisme australien en métaphysique. Mulligan et al se sont inspirés pour leur part de certaines conceptions de Husserl, Russell et Wittgenstein pour proposer une notion similaire dans leur fameux texte de 1984. Leur but était de renouer avec l'investigation de l'ontologie de la vérité entreprise par ces auteurs afin de fournir, par-delà le "tournant sémantique » de Tarski et le déflationnisme, une "élucidation de la relation basique de vérité elle-même» (voir Mulligan et al (1984) dans L \& R, p. 60) sous la forme d'une «théorie correspondantiste philosophiquement non neutre»(ibid.) susceptible de compléter la théorie purement sémantique de Tarski. Or une telle théorie exige pour son établissement l' «examen direct» du lien entre vériporteurs, le «matériel de la logique ${ }^{14}$, et vérifacteurs, qui sont "dans le monde, ce en vertu de quoi les phrases ou les propositions sont vraies" (ibid., p. 60).

Mulligan et al consacrent une bonne partie de leur texte à présenter et à examiner l'hypothèse selon laquelle une classe d'entités, les «moments »15, seraient les mieux habilitées à jouer le rôle de vérifacteurs - sans aller toutefois jusqu'à soutenir que tous les vérifacteurs seraient des moments (ainsi, pour certains vériporteurs spéciaux, tels que les énoncés d'existence ou d'identité, les trois auteurs envisagent l'hypothèse d'admettre également les choses elles-mêmes en tant que vérifacteurs). "Un moment est un objet existentiellement dépendant ou non auto-suffisant, c'est-à-dire un objet « spatiotemporel» qui est d'une nature telle qu'il ne peut pas exister seul, mais requiert l'existence d'[au moins, voir ibid., p. 64] un autre objet [appelé "fondement» ("fondament»), voir ibid., p. 64] en dehors de lui-même» (ibid., p. 61-62), dont il dépend pour son existence de manière essentielle (ibid., p. 64). Ces objets sont des particuliers, par exemple «la blancheur de

14. Notons que chez Mulligan et al, les vériporteurs sont des phrases (atomiques) plutôt que des propositions.

15. Terme emprunté à Husserl (voir ibid., p. 64). Le terme «trope» est plus généralement utilisé (y compris par Mulligan, Simons et Smith dans leurs textes ultérieurs). 
Socrate » (voulant dire cette blancheur de Socrate à tel moment précis) ${ }^{16}$. Les biconditionnels tarskiens de la forme:

Ce cube est blanc» si et seulement si ce cube est blanc ${ }^{17}$.

seront donc reformulés comme suit:

$\mathrm{Si}$ «ce cube est blanc » est vrai, alors cela est vrai en vertu de l' «être blanc» (de la blancheur) de ce cube, et si aucune blancheur de cette sorte n'existe, alors «ce cube est blanc» est faux (ibid., p. 66).

Selon les trois auteurs, le gros avantage des moments est qu'ils sont, comme les choses elles-mêmes, des objets susceptibles d'être rencontrés et perçus, et donc plus fermement ancrés dans notre ontologie et notre épistémologie que certaines entités spéciales non objectuelles postulées par d'autres théoriciens pour jouer le rôle de vérifacteurs, comme les états de choses ou les faits (voir ibid., p. 70-74). L'autre avantage des moments est qu'ils constituent pour les phrases atomiques prédicatives des vérifacteurs plus fins et mieux ciblés que les seuls objets indépendants ${ }^{18}$, d'une manière plus naturellement en accord avec la perception réelle des agents (voir la discussion intéressante sur cette question, p. 72-74). Cet argument est important pour les trois auteurs, car leur but, exposé très clairement à la fin du texte, est de "développer une théorie de la relation de vérifaction qui fasse uniquement appel à des objets fermement en lien avec notre expérience quotidienne et scientifique. Car c'est dans une telle expérience, et non dans les modèles abstraits de la sémantique logique, que se situent les origines de la connaissance de la vérité et de la fausseté » (ibid., p. 84). D'où leur critique de l'isomorphisme wittgensteinien. Ils soutiennent ainsi le principe de «l'hétérogénéité de la logique et de l'ontologie» (ibid., p. 77):

[1]a structure des objets qui rendent une phrase vraie n'est pas [...] quelque chose qui peut être dégagé de la phrase elle-même par des moyens purement logiques (ibid., p. 74).

C'est pourquoi la nominalisation, si elle peut nous mettre sur la voie en nous permettant de dégager les vérifacteurs de nombreuses phrases

16. La classe des moments englobe par ailleurs un bon nombre d'autres sortes d'entités: les frontières, les configurations et les reconfigurations "qui nécessitent un médium " comme un sourire dans un visage, un nœud dans une corde, "et plus généralement, tous les événements, les actions, les processus, les états et les conditions impliquant essentiellement des choses matérielles" (ibid., p. 62-63).

17. Notons que le «biconditionnel tarskien» utilisé ici comme exemple par Mulligan et $a l$ réfère à un objet en particulier («ce cube»), lui-même fondement d'un trope plutôt que d'un universel - et non pas à quelque chose de général (comme «la neige» chez Tarski). Comme tel, il s'inscrit dans le cadre de ce qu'on pourrait décrire comme une théorie particulariste de la vérité.

18. Ou encore des objets considérés en tant qu'ils tombent sous tels ou tels prédicats ou exemplifient tels ou tels universaux. 
simples, ne doit pas être considérée comme une méthode infaillible, ni surtout comme une élucidation substantielle de la relation de vérifaction (voir ibid., p. 66).

Mulligan et al proposent à la fin de leur texte l'ébauche d'une théorie formelle de ladite relation. Deux types de contraintes pèsent sur cette théorie: celles posées d'une part par les exigences d'une sémantique réaliste, et d'autre part, par le "principe de l'hétérogénéité de la logique et de l'ontologie» déjà mentionné:

[n]ous partirons par conséquent du principe que les relations (ontologiques) entre vérifacteurs - avec au premier chef les relations de partie et de tout sont distinctes des relations logiques entre propositions ou phrases (ibid., p. 77).

La formalisation permet effectivement à Mulligan et al. de préciser de manière intéressante les rapports de partie à tout entre vérifacteurs. Mais si le premier principe de la vérifaction («ce qui est rendu vrai est vrai ${ }^{19}$ ) semble acceptable (dans la mesure du moins où l'on accepte la relation de vérifaction en tant que telle comme relation transcatégorielle), quid de la validité du principe inverse ? Selon les auteurs la première version de ce principe $(p \exists a \cdot a \mid=p)$ est problématique parce qu'il implique l'existence d'un seul vérifacteur. Mais sa généralisation à $p \rightarrow \exists \Gamma$. $\Gamma \mid=p$ ne l'est pas moins. Car on peut s'interroger dans un cas comme dans l'autre sur l'usage que font les auteurs du symbole de l'implication logique entre un vériporteur et l'existence de son ou de ses vérifacteurs. C'est une chose de dire qu'une ou des entités rendent un vériporteur vrai; c'en est une autre d'affirmer qu'un vériporteur vrai implique logiquement l'existence d'une ou de plusieurs entités qui le rendent vrai. Peut-on soutenir un tel principe sans retomber dans l'erreur consistant à soumettre l'ontologie à la logique? Et n'est-ce pas la raison pour laquelle l'admission d'un tel principe (maximaliste) conduit à des problèmes, en particulier celui posé par les vériporteurs négatifs? D’ailleurs Mulligan et al. seront forcés de renoncer à ce principe dans son entière généralité, comme l'exprime $p \leftrightarrow \exists \Gamma . \Gamma \mid=p$.

J'ai déjà touché un mot des critiques de Greg Restall. Celui-ci a examiné dans son influent article de 1996 les "propriétés plus sombres» (Restall, 1996, p. 89), dans le contexte de la vérifaction, de l'implication classiquement comprise comme une implication stricte (selon laquelle il est nécessaire que $B$ soit vrai si $A$ est vrai). Il faut dire qu'il était d'usage, avant la parution de l'article en question, de comprendre la relation de nécessitation entre vérifacteur et vériporteur comme une implication de cette sorte, puisque, comme le souligne Restall, ladite relation «relie implication et

19. C'est-à-dire: $a \mid=p$. $\rightarrow p$, et, dans les cas où le vérifacteur n'est pas un seul objet $a$ mais une pluralité d'objets, symbolisée par une lettre grecque majuscule: $\Gamma \mid=p . \rightarrow p$. 
nécessité» (Restall, 1996, p. 89) ${ }^{20}$. Or la thèse d'implication classique conduit, non seulement à soutenir que toute vérité nécessaire est rendue vraie par n'importe quel vérifacteur, mais, conjointe avec la thèse de disjonction (selon laquelle $s$ est un vérifacteur pour $A$ ou $B$ si et seulement si $s$ est un vérifacteur pour $A$ ou si $s$ est un vérifacteur pour $B$ ), elle a pour conséquence que tout vérifacteur rend vraie toute vérité tout court.

Restall tente de pallier ces défauts en suggérant d'analyser la vérifaction en termes d'implication pertinente plutôt que classique ${ }^{21}$. Mais cette suggestion ne vaut que si l'on continue à comprendre la relation de vérifaction comme une relation d'implication, ce que justement elle n'est pas. Dire que " $A$ implique réellement $B$ si et seulement si, dans tout monde $\mathrm{W}$, tout vérifacteur de $A$ est un vérifacteur de $B$ » (Restall, 1996, p. 95) permet certes de limiter le nombre des vérifacteurs de $\mathrm{A}$ à ceux de $\mathrm{B}$ (et ainsi d'empêcher que tout vérifacteur sans restriction rende vraie toute vérité), mais ne nous apprend rien sur ce qui fait que ces vérifacteurs-là sont ceux de A et de B. On se retrouve dans une situation où c'est la vérifaction qui sert à élucider et à spécifier la relation d'implication pertinente, plutôt que le contraire ${ }^{22}$. Dans sa postface de 2009 (dans laquelle il s'emploie à "sauver » la thèse de disjonction contre les objections de Read (2000) à travers l'examen d'un problème qui a trait aux futurs contingents $\left.{ }^{23}\right)$, Restall mentionne en passant la «connexion" qu'il devrait y avoir entre un objet (ou une circonstance) $a$ et une proposition $<\mathrm{p}>$ pour que $a$ rende $<\mathrm{p}>$ vraie autrement que «faiblement ", c'est-à-dire au sens de l'implication classique (selon laquelle $<$ p $>$ est inévitable pour $a$ si et seulement si dans tout monde où $a$ existe, $\langle\mathrm{p}\rangle$ est vraie) (voir Restall, 2009, p. $101^{24}$ ). Mais qu'est-ce au juste que cette

20. Ainsi (s étant un objet quelconque du monde et $A$ une vérité) «pour tout $A, s$ est un vérifacteur de $A$ si et seulement si $s$ existe, et il est impossible que $s$ existe sans $A$ " (Restall, 1996, p. 89).

21. Conformément à une suggestion au demeurant assez vague de Bigelow, selon laquelle "chaque fois que quelque chose est vrai, il doit y avoir quelque chose dont l'existence implique de manière appropriée que cela est vrai " (Bigelow, 1988, p. 126, l'italique est de moi).

22. Comme l'admet d'ailleurs Restall dans la note 12 de son texte: "la tâche ici est de montrer que même les partisans les plus ardents des doctrines classiques peuvent comprendre l'implication pertinente, par le biais de modèles de mondes composés de vérifacteurs » (Restall, 1996, p. 97, note 12).

23. Notons qu'il y a une erreur de transcription dans le recueil de Lowe et Rami. La formule $(B)$ p. 99 doit se lire: $"(s+r) \forall p$ and $(s+r) \forall q »$. Voir le même texte (p. 2$)$ sur le site de Greg Restall: [en ligne: http://consequently.org/papers/ten2008.pdf.

Le signe $\mid=$, qui veut dire «rend vrai(e) que », a été introduit par Mulligan, Simons et Smith, 1984 (voir, p. 77). Restall l'interprète comme "est un vérifacteur pour " (voir Restall, 1996, p. 89). Armstrong utilise pour sa part le signe $\rightarrow$ (voir Armstrong, 2004b, p. 120).

24. Restall fait une remarque similaire qui résume bien sa position dans un article paru antérieurement: " [1]a vérifaction est une relation entre objets, d'une part, et assertions, d'autre part. Un objet rend une assertion vraie seulement quand l'existence de l'objet implique la vérité de l'assertion. Dans «Truthmakers, Entailment and Necessity », j'ai soutenu que cette implication devait être interprétée comme une implication pertinente. Cela veut dire que pour que $A$ 
«connexion", qui seule pourrait assurer que la relation de vérifaction soit «forte» ou "robuste» (au sens de l'implication pertinente)? Restall fait allusion un peu plus bas à l'importance que prend ici la manière dont l'objet (ou l'événement) est constitué, une variation dans la constitution pouvant faire que ce qui rende en fin de compte $<\mathrm{p}>$ vraie robustement ou pertinemment diffère selon les cas, et donc que ce ne serait pas la seule existence de l'objet ou de l'événement qui serait en cause (bien que cette existence soit nécessaire chez Restall pour la vérifaction robuste), mais en outre quelque chose de plus localisé dans la constitution de l'objet ou de l'événement pris en tant que tout, comme par exemple la victoire d'un coureur à la fin d'une course. Cette indication pourrait constituer une piste. Mais Restall n'en dit pas plus dans l'article en question. Rappelons (très brièvement) que dans l'ontologie de Restall (voir Restall, 2000, article dans lequel il propose un modèle formel de la vérifaction), les vérifacteurs sont des parties (appelées «régions») d'un monde. Celui-ci est figuré sous la forme d'un plan euclidien grillagé infini dans toutes les directions. Les carrés composant cette grille peuvent être habités ou inhabités. Une région est une portion de la grille comportant un certain nombre de carrés (habités et/ou inhabités). Ces régions sont ordonnées par inclusion suivant une contrainte fondamentale, celle de la condition d'hérédité:

si $r$ est contenue dans $s$ alors tout ce que $r$ rend vrai est aussi rendu vrai par $s$. La vérité s'étend à mesure que les régions s'étendent (Restall, 2000, p. 216).

Cette condition règle la vérifaction faible. L'idée sera donc de restreindre de manière appropriée la vérité (robuste) à une région ${ }^{25}$ :

Premièrement, nous voulons que les mondes rendent $A \Rightarrow B$ vraie seulement quand toute région dans laquelle $A$ est vrai est une région où $B$ est vrai. C'est ce qui fera de $\Rightarrow$ une implication pertinente. Deuxièmement, nous voudrions permettre aux régions d'être en quelque sorte plus discriminantes que les mondes lorsqu'elles rendent les implications vraies. Les régions peuvent être, de façon appropriée, locales (ibid., p. 223).

Pour atteindre ce but, Restall introduit la notion de restriction (des régions à des sous-régions):

Étant donné les régions $s$ et $r$, la restriction de $s$ à l'étendue de $r$ est la région $\mathrm{s} /$, c'est-à-dire la sous-région de $s$ correspondant uniquement à l'étendue de $r$ (ibid., p. 223).

$\Rightarrow B$ soit valide, il doit (au minimum) y avoir une connexion quelconque entre $A$ et $B$. Une implication n'est pas donnée simplement parce que le conséquent $B$ est nécessaire, ou parce que l'antécédent $A$ est impossible» (Restall, 2000, p. 212).

25. Ce qui n'est pas sans rappeler la notion de «vérifacteur minimal» mise de l'avant notamment par Armstrong: un vérificateur minimal pour $p$ est un vérificateur qui ne contient aucune partie propre qui serait également un vérificateur pour $p$. Le vérifacteur maximal est le monde. 
L'implication pertinente se définira dès lors comme suit:

$A \Rightarrow B$ est vrai à $r$ seulement quand, [dans toute région $s$ ] où $A$ est vrai, $B$ est vrai à la restriction de cette région à $r$ (ibid., p. 224).

L'existence sera également définie de manière relative:

Relativement à $r$, [la région] $s$ existe (ou est actuelle) seulement quand $s$ est une partie de la région $r$ [autrement dit, $s \subseteq r$, et dans ce cas, ${ }^{\mathrm{s}}{ }_{r}=s$ et $\mathrm{r}_{s}=s$ ]. [...] Si $s$ existe relativement à $r$, alors $s$ existe relativement à n'importe quelle région plus large (ibid., p. 217).

Par conséquent, la vérifaction robuste s'exprimera comme suit:

Relativement à $r, s$ rend $A$ vraie (avec succès) seulement quand $s$ est une partie de $r$ [autrement dit, quand $s$ existe], et [quand] $s$ rend effectivement $A$ vraie [autrement dit, quand $A$ vaut ( holds») dans s]» (ibid., p. 225).

On peut toutefois se demander si, dans le cadre d'une ontologie aussi atomisée (dans laquelle les objets (et les événements) ne sont que des régions, elles-mêmes sous-divisées en régions, etc., comme semble-t-il autant d'éléments indépendants), la relation de vérifaction s'en trouve véritablement éclairée, particulièrement pour les propositions prédicatives contingentes. Même si la co-validité de $\mathrm{A}$ et de $\mathrm{B}$ relativement à une région était plus à même d'assurer la pertinence de la relation d'implication entre eux deux que leur seule co-validité relativement à un ou des mondes, qu'est-ce qui fait que $A$ « vaut» dans $s$ ? Nous nous retrouvons face au problème de l' " implication transcatégorielle », toujours pas réglé. Au final, la connexion promise paraît n'être qu'un «mapping» plus fin «entre régions [ou sous-régions] et phrases» (voir ibid., p. 216). L'analyse de la relation de vérifaction en termes d'implication (même "pertinente") échoue puisque l'implication en question ne joue à proprement parler qu'entre propositions (entre $A$ et $B$ ); alors que la vérifaction en tant que telle résulte, au mieux, de ce qu'on pourrait décrire comme une sorte de survenance régionale, étant donné la validité de $A$ dans $s$ d'une part, et l'existence de $s$ relativement à $r$ d'autre part, ce qui ne constitue pas, à mon sens, une explication suffisante de la relation entre les deux ${ }^{26}$. Et comme John Heil le fait observer:

[s]i nous savons seulement que les A surviennent sur les B, nous savons seulement que les A co-varient avec les B. Il est possible qu'il en soit ainsi parce que les A sont des B, par exemple, ou parce que les B causent les A (ou parce que les $\mathrm{A}$ et les $\mathrm{B}$ ont une cause commune), ou parce que les $\mathrm{A}$ sont composés de B. À moins que nous ne soyons en mesure de jeter quelque lumière sur ce qui fonde l'invocation à la survenance, celle-ci ne fait guère que reformuler le principe de vérifaction (Heil, 2000, p. 236).

26. On peut certes imaginer (Restall demeurant silencieux à ce sujet) que $A$ soit «à propos» de $s$. Mais une remarque aussi vague ne jette pas beaucoup de lumière sur le lien spécifique entre vériporteur et vérifacteur nécessité par la relation de vérifaction. 
Au contraire de Restall, David Armstrong insiste dans sa présentation de la théorie "générale» de la vérifaction pour distinguer d'emblée clairement la relation de vérifaction de la relation d'implication, ce qu'il ne faisait pas auparavant. Il est d'ailleurs probable que la lecture (entre autres) de l'article de Restall paru en 1996 ne soit pas étrangère à cette clarification. Mais comme le remarque Horwich, "invoquer des notions restreintes de "nécessitation*" et d' "implication*", [...] est, bien entendu, simplement reconnaître le problème; cela ne constitue pas même le début d'une solution» (voir Horwich 2008, p. 199, note 10). Ainsi, selon Armstrong, la relation de vérifaction:

1) est transcatégorielle, s'établissant entre une vérité (une proposition vraie) et une portion de réalité, c'est-à-dire une entité ou des entités existant de manière indépendante dans le monde, et dont l'existence «rend» cette vérité vraie;

2) n'est pas une relation causale: « «rendre» n'est bien évidemment pas entendu ici au sens causal. La meilleure formulation de ce terme semble être fournie par la phrase "en vertu de ». C'est en vertu de cette réalité indépendante que la proposition est vraie» (Armstrong, 2004b, p. 116);

3) est une relation de nécessitation absolue ou "métaphysique " (voir Armstrong, 2004d, dans MO 2004, p. 136): si le vérifacteur existe, alors la proposition est vraie. L'argument d'Armstrong est le suivant: si un vérifacteur $T$ d'une proposition $p$ pouvait exister sans que $p$ soit vrai, alors il faudrait bien envisager que des conditions supplémentaires soient ajoutées pour que $p$ soit vrai - soit une entité supplémentaire $U$ (et alors le vérifacteur de $p$ serait $T+U$ ), soit une proposition supplémentaire $q$ (et alors le vérifacteur de $p$ serait celui de $q$ ). Moralité: nous arriverons toujours en fin de compte à identifier un vérifacteur qui nécessite la vérité de $p$. Cette conclusion vaut si l'on accepte — comme Armstrong - la thèse du maximalisme (pas de vérité sans vérifacteur), une thèse qu'Armstrong réaffirme tout en admettant ne pas avoir d'argument direct à présenter en sa faveur (voir Armstrong, 2004b, p. 117).

4) Et cette nécessitation, justement dans la mesure où elle est transcatégorielle, ne peut pas être une implication, quelque forme qu'elle puisse prendre, puisque les «termes d'une implication doivent être des propositions» (voir Armstrong, 2004b, p. 116).

Notons qu'Armstrong considère que l'expression «en vertu de» constitue la "meilleure formulation" et non pas la «meilleure explication" de la relation de vérifaction. Cette formulation permet simplement de bien marquer le fait que la relation en question n'est pas une relation causale. L' «explication» (si on veut) vient plus tard dans la section, quand Arms- 
trong spécifie que, pour être une relation de nécessitation, la relation de vérifaction doit être une relation interne (voir Armstrong, 2004b, p. 119, section 3.3 et p. 133, note 3), c'est-à-dire une relation nécessitée étant donné ses termes mêmes. Étant donné a) tel objet réel, et b) telle proposition ${ }^{27}$, dans cet ordre, "alors la relation de vérifaction (ou de falsifaction) est automatiquement déterminée, fixée, nécessitée» (ibid., p. 119). Armstrong insiste bien sur le fait qu'une telle relation n'est pas une entité supplémentaire: puisqu'elle est interne, la relation survient nécessairement, dans chaque cas, sur ses termes - ce qui constitue selon lui un «repas gratuit» au plan ontologique. Comme le résume bien Marian David:

la doctrine du repas ontologique gratuit d'Armstrong est basée sur sa notion de survenance: une entité $Y$ survient sur une entité $X$ si et seulement s'il est impossible que $\mathrm{X}$ existe et que $\mathrm{Y}$ n'existe pas, c'est-à-dire si et seulement si $\mathrm{X}$ implique ontologiquement $\mathrm{Y}$, c'est-à-dire si et seulement si l'existence de $\mathrm{X}$ nécessite l'existence de Y (David, 2005, p. 146).

\section{Survenance et/ou correspondance?}

Remarquons tout de suite qu'Armstrong parle lui aussi de «survenance » (il le fait explicitement dans Armstrong, 2010, p. 65). Certes, il s'agit d'une survenance locale, "en termes de vérifacteurs individuels pour des vérités individuelles» (Armstrong, 2004b, p. 118); et si la relation de vérifaction survient sur ses termes, c'est en vertu (sans doute) de la correspondance entre les termes en question (Armstrong n'est pas plus explicite). Alors que la survenance au sens de Bigelow (et de Lewis) est une survenance globale (sur la population des mondes et/ou, pour le second, la manière dont ceux-ci sont arrangés ${ }^{28}$ ) de la vérité sur l'être qui ne fait (surtout) pas appel à la notion de correspondance ${ }^{29}$. Et c'est ici que se situe la difficulté pour la position d'Armstrong. Autrement dit, Marian David a raison de voir un problème chez Armstrong dans sa tentative de concilier déflationnisme et correspondance (voir David, 2005, p. 146-149) — ce qui n'est pas la même chose que le désir d'enrichir la théorie tarskienne par une théorie de la vérité-correspondance, comme cherchent à le faire Mulligan et al (voir supra). Avec sa doctrine du «repas ontologique gratuit », Armstrong semble en effet soutenir, à la fois, que les entités survenantes (c'est-à-dire, en l'occurrence, la relation de vérifaction) importent et n’importent pas ontologi-

27. Pour Armstrong, les vériporteurs sont des propositions, mais des propositions situées dans le monde, et non pas dans un quelconque ciel platonicien. Elles sont les «objets intentionnels» des pensées, des croyances et des énoncés. Certes, les vérifacteurs ne peuvent pas nécessiter des pensées, des croyances ou des énoncés en tant que tels; ce qu'ils nécessitent sont des propositions, c'est-à-dire des "objets intentionnels possibles" (voir Armstrong, 2004b, p. 124-125).

28. Voir Lewis, 2001a, p. 112, section 4.

29. Voir Lewis, 2001b. 
quement, et que la vérité (qui elle-même survient sur la relation en question) est et n'est pas une propriété authentique (c'est-à-dire un universel, selon Armstrong) (voir David, 2005).

Lewis a sur la question un tout autre point de vue. Il adhère sans ambiguïté à la conception déflationniste, selon laquelle parler de vérité est une manière d'abréviation pour l'énumération potentiellement infinie de biconditionnels du type « «p» est vrai $(e)^{30}$ ssi $p »$. Ainsi, (pour reprendre un des exemples préférés de Lewis) dire que « les cochons volent» est vrai, c'est simplement dire que les cochons volent. Il n'est par conséquent nul besoin de théories substantielles telles que la théorie de la vérité-correspondance, avec leurs thèses générales sur la nature de la vérité. Non que ces théories soient incompatibles avec la conception déflationniste: car, puisque celle-ci dit "tout ce qu'il y a à dire "sur la vérité, les théories «substantielles" portent en fait sur tout autre chose que la vérité, par exemple sur le vol des cochons - en somme, sur les conditions d'existence ou la manière d'être de choses quelconques dans des mondes quelconques ${ }^{31}$. Ainsi, si l'idée à la base des théories correspondantistes (et notamment du vérifactionnisme) selon laquelle la vérité dépend du monde, semble néanmoins intéressante à Lewis, il s'autorise - étant donné que le principe de vérifaction s'applique, dans le cas des propositions contingentes ${ }^{32}$, de manière contrefactuelle - à reformuler ledit principe de manière à exprimer cette "dépendance " strictement en termes de différence entre mondes possibles. Selon Lewis, par conséquent, dire que l'existence d'un vérifacteur nécessite (la vérité d')une proposition contingente, c'est dire que dans tout monde où le vérifacteur en question existe, alors la proposition est vraie. Ainsi, le principe de vérifaction devient:

(VF) Pour toute proposition $P$ et tout monde $W$, si $P$ est vraie dans $W$, [alors] il existe quelque chose $T$ dans le monde $W$ tel que pour tout monde $V$, si $T$ existe dans $V$, alors $P$ est vraie dans $V$.

Ce qui implique logiquement que:

pour tous mondes $W$ et $V$, si une proposition $P$ est vraie dans $W$ mais pas dans $V$, alors quelque chose $T$ existe dans $W$ mais pas dans $V$ (ibid., p. 106)

Le principe de vérifaction peut dès lors être transformé en un principe plus faible de survenance à la Bigelow (voir ibid., p. 110), celui de la «différencifaction ${ }^{33}$ entre mondes. Dans la version lewisienne de ce principe,

30. Selon qu'il s'agisse d'un énoncé ou d'une proposition.

31. Pour une critique de la manière assez cavalière dont Lewis (2001b) rejette plus particulièrement la théorie correspondantiste en tant que théorie substantielle de la vérité, voir David 2004. Dans le présent article, Lewis rejette toutes les théories substantielles.

32. Car le principe de vérifaction s'applique de manière triviale, comme on l'a vu, aux propositions nécessaires (voir Restall, 1996).

33. Si je puis me permettre cette expression barbare pour traduire «difference-making»! 
ladite différence se marque, soit en termes d'existence ou d'absence de quelque chose dans un monde et pas dans un autre, soit en termes de possession, par une chose, d'une propriété, ou encore de l'entretien d'une relation entre deux choses dans un monde et pas dans un autre. Le principe de vérifaction est donc reformulé par Lewis comme suit:

(VF=) Pour toute proposition $P$ et tous mondes $W$ et $V$, si $P$ est vraie dans $W$ mais pas dans $V$, alors ou bien il y a quelque chose qui existe dans un des mondes mais pas dans l'autre, ou bien un n-tuple ${ }^{34}$ de choses entretient une relation fondamentale dans un des mondes mais pas dans l'autre (ibid., p. 112).

Dans une telle conception, nul besoin d'entités supplémentaires comme les états de choses, les faits négatifs ou généraux, etc., pour servir de vérifacteurs. Nul besoin non plus de se prononcer sur le poids ontologique de la vérité qui survient ainsi sur l'être ou sur les manières d'être; car, conformément à la doctrine déflationniste, la vérité n'est ici qu'une manière de «faire une histoire courte». Dire que «la rose est rouge» est vrai [sous-entendu: de manière contingente dans le monde actuel (par exemple)], est une manière simple et commode de dire que la rose instancie le rouge dans ce monde-ci mais qu'il serait possible qu'elle ne l'instancie pas dans tel ou tel autre monde où elle pourrait par exemple être blanche. Ce faisant, la relation de vérifaction proprement dite disparaît. Lewis parle bien à un certain moment "d'implication» (seulement en ce qui concerne les propositions nécessaires, voir ibid., p. 104), mais autrement toute mention de ce terme, de même que toute référence à une quelconque nécessitation, sont évacuées au profit d'une simple co-variance entre l'être et la vérité d'un monde à un autre: une "vérifaction", somme toute, sans vérifacteurs ni (relation de) vérifaction.

Cette manière de reformuler le principe de vérifaction a été critiquée, notamment par Armstrong (voir Armstrong, 2004b, p. 118) et RodriguezPereyra (voir Rodriguez-Pereyra, 2005, p. 228-229). Pour l'un comme pour l'autre, la reformulation de la vérifaction en termes de survenance à la Bigelow ou à la Lewis n'est pas fausse, elle est incomplète. Selon Armstrong, une telle conception ne rend pas justice à l'intuition à la base du principe de vérifaction, qui commande une approche parcellaire [piecemeal $]^{35}$ et particulière concernant la vérité - des vériporteurs individuels, pour des vérifacteurs individuels (même s'il ne s'agit plus d'une correspondance terme à terme) - dont le but est (si possible) l'identification, pour chaque vériporteur, de son vérifacteur minimal. De même, Rodriguez-Pereyra insiste sur le fait que la relation de vérifaction n'est pas réductible à la survenance, bien

34. Lewis précise que les 1-tuples sont équivalents aux choses elles-mêmes, ce qui permet de couvrir les cas où la différence d'un monde à l'autre a trait aux propriétés intrinsèques d'une chose (ibid., p. 112).

35. voir Armstrong, 2004b, p. 118. 
que la seconde s'ensuive de la première. Son argument principal est que la survenance est une relation symétrique, alors que la vérifaction est une relation asymétrique (la vérité étant fondée dans la réalité, mais non le contraire).

L'opposition Armstrong-Lewis est mieux comprise si on la replace dans un contexte plus large, qui est celui de l'admission ou du rejet de connexions qu'il faut bien appeler nécessaires. Car, comme l'a bien vu David Lewis: "demander des vérifacteurs, c'est demander des connexions nécessaires, purement et simplement» (Lewis, 1998, p. 219).

Ces connexions sont:

1) les connexions entre vériporteurs et vérifacteurs dans la relation de vérifaction, du moment qu'elle est conçue comme une relation de nécessitation;

2) les connexions au sein même des entités postulées pour jouer le rôle de vérifacteurs (comme les états de choses) ${ }^{36}$ - lesquelles connexions doivent, en fin de compte, être considérées peu ou prou comme nécessaires, ne serait-ce qu'a posteriori, parce que la nécessitation en jeu dans la relation de vérifaction est elle-même fonction de la «nécessité " de ces connexions. Lewis a d'ailleurs remarqué - avec justesse - un conflit chez Armstrong entre combinatorialisme (entendu comme théorie combinatoire de la possibilité) et vérifaction ${ }^{37}$. Ce conflit est sous-jacent au conflit signalé par Marian David entre déflationnisme et correspondance chez Armstrong (voir supra).

Les critiques du vérifactionnisme (comme Lewis, et à sa suite, Hornsby et Dodd):

1) adhèrent au principe humien de recombinaison, selon lequel n'importe quelle chose peut coexister avec n'importe quelle autre, pourvu du moins qu'elles occupent des positions spatio-temporelles distinctes" (Lewis, 1986, p. 88) et à la doctrine de la «survenance humienne», que Lewis définit comme suit:

toute la vérité à propos d'un monde comme le nôtre survient sur la distribution spatio-temporelle des qualités locales (1986, p. ix-xvi). [...] [T]oute la vérité contingente survient simplement sur la structure de co-instantiation, peu importe les «crochets » particuliers auxquels sont suspendus les liens et les relations (Lewis 1994, p. 473-474).

36. Ou alors, connexions sous forme de dépendance entre fondement et moments, substance et tropes, ou encore au sein d'un faisceau de tropes ou d'universaux.

37. Voir Lewis, 1998, p. 220. Voir aussi Kistler, 2004. 
2) conçoivent la vérité en termes de survenance globale, d'une manière qui s'oppose à la conception parcellaire des vérifactionnistes;

3) reprochent aux vérificationnistes d'enfreindre l'interdit humien des connexions nécessaires.

Notons en outre que le clivage entre les vérifactionnistes et leurs critiques se marque sur le plan de l'engagement ontologique chez les uns et chez les autres. Les vérifactionnistes s'engagent à l'existence d'entités composées et complexes (par exemple les états de choses), alors que leurs adversaires prétendent limiter leur ontologie (comme le préconisait Quine) aux seules choses matérielles. Dans une telle ontologie, les propriétés se réduisent à marquer de "simples différences qualitatives" entre les mondes (Dodd, 2002, p. 80), ou entre différents (ré)arrangements dans le monde.

Les vérificationnistes (en particulier Simons ${ }^{38}$ ) ont pu rétorquer à leurs critiques qu'une conception à la Mulligan et al. selon laquelle les vérifacteurs sont des entités dépendantes, ne contrevient pas à la survenance humienne. Armstrong soutiendra un argument similaire au sujet des connexions (nécessaires) entre les particuliers et les universaux dans les états de choses: étant donné que les particuliers et les universaux composant les états de choses sont partiellement identiques (voir Armstrong, 2004e), l'interdiction humienne des connexions nécessaires entre entités distinctes n'est pas transgressée. Grâce à cette notion d'identité partielle ${ }^{39}$, Armstrong peut ainsi sauter le pas et finalement reconnaître que «les particuliers possèdent leurs propriétés de manière nécessaire ", ce qui lui permet de fonder la relation de vérifaction sur des bases plus solides, en même temps que de couper court au reproche de Lewis mentionné ci-dessus (selon lequel il y aurait, chez Armstrong, conflit entre combinatorialisme et principe de vérifaction). Mais n'anticipons pas ${ }^{40}$. Le fait est que dans «The General Theory of Truthmaking", le texte d'Armstrong qui nous occupe ici, la relation de vérifaction présentée en termes de survenance locale ne s'en trouve pas beaucoup plus éclairée que dans sa présentation (ou plutôt, sa reformulation) en termes de survenance globale.

\section{5. «The Current Debate»}

La troisième partie du recueil de Lowe et Rami propose un échantillon intéressant des discussions plus récentes sur la nature et la justification de la relation de vérifaction. Deux articles, notamment, sont dignes d'attention,

38. Voir par exemple Simons, 1994 et 2000.

39. Inspirée par une suggestion de Baxter, 2001.

40. Ce n'est pas ici le lieu pour exposer en détail ce changement de cap important chez Armstrong, et ses conséquences sur sa métaphysique. Pour une discussion de ces questions, voir certaines remarques de E. J. Lowe (Lowe, 2009, p. 210-211); voir aussi Svennerlind, 2005, et Armstrong, 2007. 
parce qu'ils permettent de poursuivre et d'approfondir le débat amorcé dans la partie précédente: celui de Rodriguez-Pereyra (paru d'abord dans Beebee et Dodd, 2005) qui critique la conception lewisienne, suivi d'une postface inédite dans laquelle il répond aux critiques de Jennifer Hornsby ${ }^{41}$; celui de Horwich, qui critique plus particulièrement la conception armstrongienne (mais aussi, plus généralement, la théorie de la vérifaction sous toutes ses formes), et auquel Armstrong lui-même a répondu (dans sa première version en français) dans Monnoyer, 2004. Marian David examine quant à lui l'idée reçue selon laquelle le principe de vérifaction serait une version améliorée de la théorie de la vérité-correspondance et que la seconde impliquerait le premier, mais pas l'inverse. David s'interroge sur le bien-fondé de cette présupposition, ne serait-ce que parce que la correspondance est une relation symétrique alors que la vérifaction est supposément une relation asymétrique (ou plutôt, non symétrique, comme le soutient plutôt David). L'article de Lowe, enfin, vaut largement le détour, car il met de l'avant une conception différente de la relation de vérifaction selon laquelle la nécessité est explicitée en termes d'essence.

Rodriguez-Pereyra se donne pour tâche de montrer, contra Lewis et al., que la thèse elon laquelle la vérité est fondée dans la réalité nous engage à la thèse selon laquelle les propositions vraies sont rendues vraies par des entités (Rodriguez-Pereyra, 2005, p. 232), et non pas simplement par la manière dont sont les choses:

Pas de vérifaction sans vérifacteurs est mon slogan. [...] mon seul but ici est de défendre la thèse selon laquelle, si une proposition est rendue vraie par quelque chose, elle est rendue vraie par quelque chose (ibid., p. 227).

Selon Rodriguez-Pereyra, ces entités seraient des faits (ou des états de choses, ce qui revient, semble-t-il, à la même chose pour lui, voir note 2), mais il n'entend pas argumenter dans le présent texte en faveur d'un type d'entités plutôt qu'un autre. Il accepte également sans discuter les propositions comme vériporteurs ${ }^{42}$. Son but est de défendre le principe de vérifaction pour au moins une classe de propositions synthétiques vraies. La question à laquelle Rodriguez-Pereyra se doit de répondre est la suivante:

Peut-on vraiment maintenir que la vérité est déterminée par la réalité sans maintenir qu'il y a des vérifacteurs? En d'autres termes, peut-on vraiment maintenir que la proposition que la rose est rouge est rendue vraie par la manière dont sont les choses, et non pas par l'existence des choses? (ibid., p. 232).

Rodriguez-Pereyra a recours à deux arguments: 1) l'argument que j'appellerai l'argument de la nécessité de la réification (ibid., p. 232-234); et

41. N.B. Le texte de Hornsby est également paru dans Beebee et Dodd, 2005.

42. Notons toutefois que ce sont des phrases qui sont appelées à jouer ce rôle dans son ouvrage d'orientation «nominaliste» de 2002. 
2) l'argument selon lequel la vérité est fondée dans des entités, et ce, parce que: a) la fondation (grounding) est une relation; et b) les relations relient entre elles des entités (ibid., p. 234-239).

Voyons d'abord le premier argument. L'idée qui sous-tend cet argument est que, non seulement la vérité est fondée dans la réalité, mais que des vérités différentes sont fondées dans différentes portions de la réalité, ou différents aspects (features) de la réalité, et donc, que «des vérités différentes portant sur la même matière sont déterminées par des aspects différents de la matière en question" (ibid., p. 233). Car la rose peut être rouge ou blanche dans différents mondes possibles; mais elle peut être aussi, dans le même monde, à la fois rouge, parfumée, veloutée, etc., bref, elle peut présenter divers aspects, posséder (ou instancier) diverses propriétés, être de plusieurs manières (ways). Celles-ci peuvent être distinguées, ce qui veut dire qu'elles peuvent être identifiées, comptées et faire l'objet d'une quantification (ibid., p. 233). Mais alors:

si on peut identifier, compter et quantifier sur des manières, alors les manières existent. Autrement dit, les manières, qui sont des vérifacteurs, existent. Nous en revenons par conséquent [au principe de vérifaction], lequel affirme que les propositions vraies sont rendues vraies par des entités, et implique que la vérité survient sur l'existence des choses (ibid., p. 233).

La réification de ces différents aspects est donc nécessaire, selon Rodriguez-Pereyra, car seule elle peut permettre de rendre compte de la vérité de chaque proposition particulière. Soutenir simplement que la vérité des propositions à propos d'une chose dépend de (survient sur) la manière (how) dont la chose est, ne permet pas d'individuer ce qui rend vrai chacune de ces propositions, chacune étant vraie en vertu de la même chose. Or cette même chose a plusieurs manières d'être.

Le deuxième argument est décomposé par Rodriguez-Pereyra en trois prémisses et une conclusion:

1. La vérité est fondée.

2. La fondation est une relation.

3. Les relations relient entre elles des entités.

4. Par conséquent, la vérité est fondée dans des entités (ibid., p. 234).

Selon Rodriguez-Pereyra, les prémisses (1) et (3) sont indéniables. À peu près tout le monde s'entend sur (1), c'est-à-dire sur le fait que la vérité est fondée dans la réalité. Quant à (3), elle exprime le fait que, tout comme les propriétés qui sont possédées ou instanciées obligatoirement par des entités, les relations s'établissent obligatoirement entre des entités (ibid., p. 234). Par conséquent, si l'on entend nier la conclusion (4) de l'argument, il faut s'attaquer à la prémisse (2). Rodriguez-Pereyra entreprend de prouver la validité de (2) en réfutant successivement six autres manières de concevoir la fondation autrement que comme une relation (ibid., p. 235-239). Je mentionnerai simplement la discussion concernant la descente sémantique telle 
que proposée par Quine, dans laquelle la fonction du prédicat de vérité n'est pas l'attribution d'une propriété, mais simplement la décitation. La descente sémantique autorise à passer du discours sur les phrases (ou les propositions) au discours sur les choses. Mais la descente sémantique n'explique pas que la vérité d'une proposition soit fonction de la réalité. C'est parce que la proposition que la rose est rouge est vraie parce que la rose est rouge qu'il est possible — du moment que la rose est rouge — d'appliquer légitimement le procédé de la décitation, pas le contraire. Autrement dit, être autorisé à passer du discours sur les phrases (ou les propositions) au discours sur les choses n'explique pas que la vérité du discours soit fonction des choses.

Rodriguez-Pereyra soutient que si la fondation est une relation, alors elle est une relation asymétrique (nous avons déjà vu cela) et que la vérité est une "propriété relationnelle des propositions " (ibid., p. 235), qui s'applique en vertu d'une relation entre ce à quoi cette propriété s'applique (c'est-à-dire une proposition) et quelque chose d'autre (c'est-à-dire l'entité sur laquelle porte la proposition en question). Si la fondation est une relation, la vérité est fondée dans des entités, et, selon Rodriguez-Pereyra:

on ne peut échapper aux vérifacteurs une fois admis le fait que la vérité requiert une fondation. Il n'est pas possible de maintenir que la vérité est fondée dans la manière dont sont les choses sans maintenir que la vérité est fondée dans l'existence des choses. Par conséquent, [le principe de vérifaction] et l'idée que les vérités ont des vérifacteurs sont légitimés (ibid., p. 239).

En ce qui concerne le premier argument de Rodriguez-Pereyra, Jennifer Hornsby critique la réification à l'origine des vérifacteurs, qui est annoncée (et en quelque sorte dictée) selon elle par la nominalisation en jeu dans des expressions comme: "the rose's being red». Rodriguez-Pereyra se défend dans sa postface d'avoir voulu faire dépendre l'ontologie du langage. Le point ici - au-delà de la question de la nominalisation - est de savoir s'il est ou non possible de rendre compte du fait que la vérité soit fondée dans la réalité sans accepter que la fondation soit une relation qui relie des entités (voir Beebee et Dodd, 2005, p. 8-9). Selon Hornsby, il est possible de concevoir, en pensant à la vérité de <la rose est rouge>, une proposition qui soit distincte de la rose et qui suffise à établir l'asymétrie exigée par Rodriguez-Pereyra sans multiplier indûment les entités. De même qu'il est possible d'expliquer en termes d'instanciation que la rose puisse être à la fois rouge et légère, ou rouge sans être légère, sans pour autant réifier les manières d'être de la rose. Nous retrouvons ici une conception similaire à celle de Lewis.

Un point plus intéressant (car ces questions n'ont pas été examinées dans la partie précédente) est celui que Jennifer Hornsby soulève en notant que Rodriguez-Pereyra parle indifféremment de la vérité des propositions comme étant fondée dans la réalité, déterminée ou expliquée par la réalité, comme étant telle en vertu de la réalité, ou parce que la réalité est telle ou 
telle. Hornsby relève ces «équivalences approximatives» et en questionne les implications dans la première partie de son texte (voir Hornsby, 2005, p. 35; Rami fait un reproche similaire dans son introduction [voir Rami, 2009 , p. 23]). Rodriguez-Pereyra nie qu'il fait une équivalence entre détermination et explication, les faits concernant la seconde dépendant de ceux concernant la première, mais reconnaît qu'il n'a peut-être pas été suffisamment clair à ce sujet (voir Rodriguez-Pereyra, 2009, p. 242-243).

En ce qui concerne le deuxième argument de Rodriguez-Pereyra, enfin, un problème à mon sens est que ledit argument laisse entendre, si les entités en question sont conçues comme indépendantes ${ }^{43}$, que la relation de fondation pourrait être une relation externe plutôt qu'interne, contrevenant au principe humien si elle est néanmoins considérée comme une relation nécessaire, et posant problème pour la vérifaction si elle n'est pas considérée comme une relation nécessaire. Selon Rodriguez-Pereyra, la thèse selon laquelle la vérité serait déterminée par la réalité est en elle-même une idée très modeste: "c'est simplement l'idée selon laquelle la vérité d'un vériporteur est déterminée par ce sur quoi elle porte ( its subject matter»), ou un de ses aspects» (Rodriguez-Pereyra, 2005, p. 231). Cette conception peut se révéler problématique si le vériporteur n'est pas une proposition.

La question du rapport entre détermination et explication est examinée par Horwich dans son article, dans une perspective qui inverse le rapport de dépendance entre les deux. Lowe vise quant à lui à fournir une explicitation de la nature du rapport entre vériporteurs et «ce sur quoi ils portent» d'une part, et vérifacteurs d'autre part, dans une perspective (voisine de celle de Kit Fine) qui inverse le rapport de dépendance entre nécessité et essence.

Horwich commence par réfuter l'idée qu'une théorie vérificationniste révèle la nature de la vérité et justifie ce faisant l'intuition selon laquelle cette nature réside dans la correspondance de la vérité avec la réalité. Car la version la plus simple de la théorie vérificationniste impliquerait qu'à chaque proposition vraie corresponde un fait qui lui soit spécifique. Ainsi, la proposition que Mars est rouge ou vert serait rendue vraie par le fait disjonctif que Mars est rouge ou vert. Mais les vérificationnistes eux-mêmes reconnaissent que l'admission de tels faits disjonctifs (comme aussi les faits négatifs, généraux, etc.) pose problème. C'est pourquoi ils ont proposé différentes versions de la théorie afin d'expliquer la vérifaction sans trop d'extravagances ontologiques. Ces différentes versions, reposant chacune sur divers principes complexes et privilégiant chacune tel ou tel type d'entités ou de faits plus ou moins artificiels, ont toutefois pour résultat que la nature de la vérité qu'elles révèlent est loin d'être simple. Il est clair, fait observer Horwich, qu'un locuteur ordinaire ne conçoit pas la vérité en ces termes. Il vaut mieux

43. Et on pourrait le croire, si l'on se reporte à la conception nominaliste de RodriguezPereyra selon laquelle les vériporteurs seraient des phrases. 
considérer par conséquent qu'une théorie vérifactionniste présuppose la compréhension du concept de vérité, plutôt qu'elle ne la fournit. Horwich en conclut que nous sommes libres d'interpréter une théorie de la vérité telle que la théorie vérifactionniste en faisant appel à la conception de la vérité la plus simple qui soit, à savoir la conception déflationniste. Selon cette conception (comme on l'a vu à propos de Lewis), la vérité n'est pas une propriété; le concept de vérité est un simple outil de généralisation, permettant de reformuler sous forme de thèse générale un nombre potentiellement infini d'énoncés. L'utilisation de cet «outil» n'implique pas que les énoncés qu'il sert à formuler soient à propos de la vérité. Par conséquent, selon Horwich, le "véritable contenu d'une théorie vérifactionniste» (en tant que prétendue théorie de la vérité) réside en fait dans des affirmations de la forme " $p$ en vertu de $x$ ». L'introduction de la notion de vérité vient après. Ainsi, on peut remplacer $" p$ " par $"<p>$ est vraie » pour obtenir $"<p>$ est vraie en vertu de $x »$, ou, autrement dit, « $\langle p>$ est rendue vraie par $x »$. On peut ensuite généraliser pour obtenir le principe de base de la vérifaction, selon lequel «toute proposition (ou encore plus généralement, tout vériporteur) est rendu(e) vrai(e) par quelque chose» (voir Horwich, 2008, p. 189). C'est pourquoi la théorie de la vérifaction n'est pas une théorie de la vérité; mais de plus, la théorie de la vérifaction ne fournit même pas une contribution valable au plan métaphysique, contrairement à ce que soutient notamment Armstrong. La thèse fondamentale de Horwich est que rien ne rend vrai (ou, plus généralement, ne rend $F$ ) quoi que ce soit. Ainsi, dans le schéma " $p$ en vertu de $x$ », "en vertu de » doit s'analyser en termes d'explication ( "parce que ») et non pas en termes de nécessitation (d'une chose (ou d'une propriété d'une chose) par une autre): " $\langle p\rangle$ est vraie parce que $p$ ", $p$ étant déduit (expliqué) à partir de lois physiques et de telles ou telles conditions initiales. Ensuite seulement l'existence du fait que $p$ peut être expliquée, à partir de $p$. En bref, "ce n'est pas à cause du fait que ["because of the fact that " ${ }^{44}$ ] Mars est rouge (ou [à cause de] l'état de rougeur de Mars [«the state of Mars being red»]) que Mars est rouge. Au contraire, c'est parce que Mars est rouge qu'un tel fait existe (et qu'un tel état est actuel)» (ibid., p. 193). Selon Horwich, donc, «[1]es faits [...] ne peuvent pas être fondamentaux du point de vue explicatif» (Horwich, 2004, p. 126). En ce sens, l'existence n'est pas basique (voir Horwich, 2008, p. 198).

Ce à quoi Armstrong rétorque: "[u]ne telle substitution du concept épistémologique d'explication à celui de vérifacteur laisse dans l'obscurité presque complète ce que [Horwich] pense de l'ontologie de la situation " (Armstrong 2004e, p. 135). En effet, d'autant plus que la critique que fait Horwich de la théorie de la vérifaction est censée valoir, comme celui-ci le

44. Horwich peut se permettre d'utiliser le même terme («because»), ce qui est plus difficile en français. 
répète à plusieurs reprises, pour les vériporteurs autres que les propositions et pour les vérifacteurs autres que les faits.

Pour E. J. Lowe, il est clair que, pour être vraie, une proposition doit être "rendue vraie" par quelque chose (un ou des vérifacteurs); qu'un vériporteur est vrai «en vertu de » son ou ses vérifacteurs; et qu'il s'agit là d'une «relation d'explication métaphysique (je souligne)» (Lowe, 2009, p. 201202). En d'autres termes, la vérifaction doit être, du même coup, une explication de la manière dont une proposition vraie "en vient à être vraie" (ibid., p. 202).

Lowe part d'un constat: l'analyse de la relation de vérifaction en termes de nécessitation est - comme on l'a vu ci-dessus — «incapable de discriminer suffisamment finement entre les vérifacteurs de propositions différentes» (ibid., p. 201). Il s'ensuit que l'expression «en vertu de» demeure inanalysée.

Lowe poursuit par une remarque: les prédicats ontologiques formels, tels que «est un trope », "est un particulier concret», "existe», "est identique à » et «est vrai», expriment des propriétés ontologiques formelles. De telles propriétés ne sont pas des "éléments de l'être", mais "contribuent à la nature de la réalité », dans sa manière d'être (ibid., p. 202). Les règles d'application de ces prédicats diffèrent de celles des prédicats empiriques ou descriptifs ordinaires: un prédicat ontologique formel s'applique à une entité "en vertu de son identité", c'est-à-dire "en vertu de ce qu'est cette entité», bref, «en vertu de son essence» (voir ibid., p. 206). Or il peut faire partie de l'essence d'une entité qu'elle dépende pour son identité ou pour son existence, de l'identité ou de l'existence d'autres entités. Aussi un trope (ou un mode, pour user de la terminologie de Lowe) dépend-il, tant pour son identité que pour son existence, de l'identité et de l'existence de la substance individuelle ou du particulier concret qui le possède. Un universel ne dépend pas essentiellement, pour son identité, de tel ou tel mode particulier qui l'instancie; mais il dépend essentiellement, pour son existence, de ses instanciations (car dans l'ontologie de Lowe, il n'y a que des universaux immanents, instanciés par des modes, qui font ainsi office de médiateurs entre les universaux et les substances).

La dépendance essentielle n'est cependant pas le seul type de dépendance métaphysique: il y a également la «simple» dépendance nécessaire. Et c'est là, selon Lowe, tout le problème de la théorie de la vérifaction orthodoxe: elle fait appel à la dépendance nécessaire plutôt qu'à la dépendance essentielle. Or un être nécessaire (comme le nombre sept, en supposant que les nombres existent et soient effectivement nécessaires) nécessite en tant que tel l'existence de n'importe quelle entité (par exemple, une pomme), bien que cette pomme ne dépende assurément pas de manière essentielle, pour son existence, du nombre sept. De manière analogue, "nous devrions dire qu'une proposition dépend nécessairement pour sa vérité d'une certaine entité seulement dans le cas où il est métaphysiquement nécessaire que la 
proposition soit vraie seulement si l'entité en question existe» (ibid., p. 208). Mais alors, "la proposition que cette pomme est ronde dépend nécessairement pour sa vérité de [l'existence] du nombre sept» (ibid., p. 208), ce qui est évidemment contre-intuitif (voir Restall, 1996).

La solution de Lowe - on l'aura deviné - est de faire appel à la notion de dépendance essentielle, et de définir un vérifacteur pour une proposition comme "une chose telle que cela fasse partie de l'essence de cette proposition d'être vraie si cette chose existe» (ibid., p. 209). Mais qu'est-ce à dire? Comment détermine-t-on que quelque chose fait partie de l'essence de quoi que ce soit? Dans le cas d'une proposition, il s'agirait, pour Lowe, de l'entité ou des entités sur lesquelles celle-ci "porterait essentiellement » et dont elle dépendrait essentiellement pour son identité (et par suite, de l'existence desquelles elle dépendrait essentiellement pour son existence) (voir p. 213$)^{45}$. Mais comment s'assurer que l'essence telle qu'on la détermine est bien l'essence telle qu'elle est? Et quid des autres types de vériporteurs, comme les phrases ou les énoncés (puisque Lowe affirme que ce qu'il dit des propositions vaut également pour eux (voir ibid., p. 201)): peut-on dire d'eux aussi qu'ils portent de manière essentielle sur ce dont ils portent ?

Quoi qu'il en soit, Lowe a le mérite de chercher à proposer une analyse plus fine du rapport entre vériporteurs et vérifacteurs. De plus, l'ajout des modes dans son ontologie lui permet de fournir une réponse au problème de la possibilité des vérités prédicatives contingentes et d'éviter de faire de toute prédication vraie une prédication nécessaire (car les modes - qui tiennent lieu de relations entre substances et universaux — sont des êtres contingents) ${ }^{46}$.

$\mathrm{Au}$ final, et malgré les réserves formulées au début de cette étude, le recueil de Lowe et Rami s'avère d'une lecture stimulante par la richesse, la profondeur et la diversité des points de vue rassemblés. Quiconque s'intéresse à la question de la vérifaction, et plus généralement aux questions touchant la théorie de la vérité, l'engagement ontologique, la dépendance métaphysique, pour ne mentionner que celles-là, tirera profit de cet ouvrage ${ }^{47}$.

45. Quoi qu'il en soit, Lowe doit nuancer sa définition du vérifacteur, car le vérifacteur d'une proposition n'est pas toujours ce sur quoi elle porte: par exemple, la proposition que la pomme est rouge porte essentiellement, selon Lowe, sur la substance individuelle "pomme » et l'universel «rouge ", mais le vérifacteur de cette proposition est le mode de la rougeur de cette pomme. Cependant, $c e$ mode aurait pu être un autre mode. Il n'entre donc pas dans l'essence de la proposition d'être rendue vraie par l'existence de $c e$ mode (qui aurait pu ou pourrait ne pas exister bien que la proposition, elle, existe), mais, plus modestement, par l'existence d'un mode de ce type (voir ibid., p. 212-215). Il semble que la définition essentialiste de la vérifaction ne permette pas de discriminer aussi finement que souhaité.

46. Voir le passage extrêmement intéressant dans lequel Lowe critique le recours aux faits comme vérifacteurs, et en particulier la conception armstrongienne (ibid., p. 210-212).

47. Merci à Claude Panaccio pour sa lecture attentive. 


\section{Bibliographie}

Armstrong, D. M. (2004a). Truth and Truthmakers, Cambridge, Cambridge University Press.

- (2004b). "The General Theory of Truth-Making ", chapitre deux de Truth and Truthmakers, dans Lowe et Rami, 2009, p. 115-133.

—. (2004c). "Vérités et vérifacteurs ", dans Monnoyer, 2004, p. 101-114.

—. (2004d). «Défense de la théorie des vérifacteurs. Une réponse à Paul Horwich », dans Monnoyer, 2004, p. 129-140.

—. (2004e). "Particulars Have Their Properties of Necessity», Pufendorf Lectures, [en ligne: http://www.pufendorf.se/2004_lecture_3.html].

—. (2007). "Revisions and Quiddities», [en ligne: http://eprints.nottingham. ac.uk/718/1/Revisions_and_quiddities.pdf].

- (2010). Sketch for a Systematic Metaphysics, Oxford, Oxford University Press.

Baxter, D. L. M. (2001). "Instantiation as Partial Identity ", Australasian Journal of Philosophy, 79, p. 449-464.

Beebee, H. et J. Dodd (dir). (2005). Truthmakers: The Contemporary Debate, Oxford, Oxford University Press.

—. (2005). "Introduction», dans Beebee et Dodd, 2005, p. 1-16.

Caputo, S. (2007). "Truth-Making: What It Is Not and What It Could Be», dans Monnoyer, 2007, p. 275-311.

Daly, C. (2005). "So Where's the Explanation?", dans Beebee et Dodd, 2005, p. 85-103.

David, M. (2004). "Don't Forget About the Correspondence Theory of Truth", Australasian Journal of Philosophy, vol. 82, $\mathrm{n}^{\circ}$ 1, p. 42-47.

—. (2005). "Armstrong on Truth-Making», dans Beebee et Dodd, 2005, p. 141159.

—. (2009). "Truth-Making and Correspondence», dans Lowe et Rami, 2009, p. 137-157.

Dodd, J. (2002). "Is Truth Supervenient on Being ? ", Proceedings of the Aristotelian Society, vol. 102, $\mathrm{n}^{\circ}$ 1, p. 69-86.

- (2010). "Truth and Truth-Making», Analysis, vol. 70, n 3, p. 567-571.

Efird, D. (2009). "Truth and Truth-Making», Notre Dame Philosophical Reviews, [en ligne: http://ndpr.nd.edu/review.cfm? id=17066].

Forrest, P. et D. Khlentzos (dir.) (2000). "Special Issue: Truth Maker and Its Variants », Logique et analyse, vol. 43, n 169-170.

- (2000). "Introduction: Truth Maker and Its Variants", dans Forrest et Khlentzos, 2000, p. 3-15.

Heil, J. (2000). «Truth Making and Entailment », dans Forrest et Khlentzos, 2000, p. 231-242.

Hochberg, H. (2009). «Facts and Relations: The Matter of Ontology and of TruthMaking ", dans Lowe et Rami, 2009, p. 158-184.

Hornsby, J. (2005). "Truth Without Truthmaking Entities ", dans Beebee et Dodd, 2005, p. 33-47.

Horwich, P. (2004). «Une critique de la théorie des vérifacteurs », dans Monnoyer, 2004, p. 115-127.

—. (2008). «Being and Truth», dans Lowe et Rami, 2009, p. 185-200.

Kistler, M. (2004). "Le combinatorialisme et le réalisme nomologique sont-ils compatibles? ", dans Monnoyer, 2004, p. 199-221. 
Lewis, D. (1986). On the Plurality of Worlds, Oxford, Blackwell.

(1994). «Humean Supervenience Debugged», Mind, New Series, vol. 103, $\mathrm{n}^{\circ} 412$, p. 473-490.

- (1998). "A World of Truthmakers?", dans Papers in Metaphysics and Epistemology, Cambridge, Cambridge University Press, 1999, p. 215-220.

—. (2001a). «Truth-Making and Difference-Making », dans Lowe et Rami, 2009, p. $102-114$.

— (2001b). "Forget About the "Correspondence Theory of Truth " ", Analysis, vol. $61, \mathrm{n}^{\circ} 272$, p. $275-280$.

Lowe, E. J. et A. Rami (dir.) (2009). Truth and Truth-Making, Stocksfield, Acumen.

Lowe, E. J. (2007) «Truthmaking as Essential Dependence», dans Monnoyer, 2007, p. 237-259.

-. (2009) «An Essentialist Approach to Truth-Making», dans Lowe et Rami, 2009, p. 201-216.

Monnoyer, J.-M. (dir.) (2004). La structure du monde, objets, propriétés, états de choses: renouveau de la métaphysique dans l'école australienne, Paris, Vrin.

- (dir.) (2007). Metaphysics and Truthmakers, Frankfurt, Ontos.

Mulligan, K., P. Simons et B. Smith (1984). "Truthmakers », dans Lowe et Rami, 2009, p. 59-86.

Mulligan, K. (2009). "Truth and the Truth-Maker Principle in 1921 », dans Lowe et Rami, 2009, p. 39-58.

Rami, A. (2009). "Introduction: Truth and Truth-Making", dans Lowe et Rami, 2009, p. 1-36.

Restall, G. (1996). «Truth-Makers, Entailment and Necessity », dans Lowe et Rami, 2009, p. 87-97.

—. (2000). «Modelling Truthmaking», dans Forrest et Khlentzos, 2000, p. 211230.

—. (2009). "Postscript to "Truth-Makers, Entailment and Necessity»", dans Lowe et Rami, 2009, p. 98-101.

Rodriguez-Pereyra, G. (2002). Resemblance Nominalism: A Solution to the Problem of Universals, Oxford, Clarendon Press.

—. (2005). "Why Truthmakers", dans Lowe et Rami, 2009, p. 227-241.

- (2009). «Postscript to «Why Truth-Makers»", dans Lowe et Rami, 2009, p. 242-250.

Simons, P. (1994). "Particulars in Particular Clothing: Three Trope Theories of Substance», Philosophy and Phenomenological Research, vol. 54, $\mathrm{n}^{\circ}$ 3, p. 553-575.

- (2000). «Identity Through Time and Trope Bundles ", Topoi, 19, p. 147-155.

Simons, P. et J. Melia (2000). "Continuants and Occurrents", Proceedings of the Aristotelian Society, Supplementary Volumes, 74, p. 59-75 et 77-92.

Svennerlind, C. (2005). "Armstrong On Instantiation As Partial Identity ", Kapten Nemos Kolumbarium, Philosophical communications, web series, $n^{\circ} 33$. 